\title{
Current Status and the Future of Spine Radiosurgery
}

\section{Arjun Sahgal ${ }^{*}$}

Department of Radiation Oncology, Princess Margaret Hospital and the Sunnybrook Health Sciences Centre, University Avenue, Toronto, Ontario, Canada

Spine Radiosurgery (SRS), also known as spine stereotactic body radiotherapy (SBRT), represents a stark departure from the treatment of spinal metastases with conventional radiation. In the past, we simply delivered a dose of radiation aimed to achieve short term pain relief without the intention of long-term local tumor and pain control. The doses of $20 \mathrm{~Gy}$ in 5 fractions, 30 Gy in 10 fractions and 8 Gy in 1 fraction are most common, and were chosen based on the limiting factor of spinal cord tolerance.

With advances in radiation technology that includes sophisticated body immobilization devices, intensity modulated radiotherapy (IMRT), image-guided radiotherapy (IGRT) and robotic technology we are now able to dose escalate spinal metastases, and deposit 2 to 6 times the biologically effective dose as compared to conventional radiation while still sparing the spinal cord to a safe dose $[1,2]$. Spine SRS is based on routinely treating with 16-24 Gy in 1 fraction, 24 Gy in 2 fractions, and 24-30 Gy in 3 fractions, with the intent to improve long-term local tumor control rates and increase the rate of both complete pain relief and long-term pain control [2,3]. The philosophical shift is in the treatment intent, as we now aim to deliver a locally "curative" dose of radiation as opposed to a locally "palliative" dose of radiation to the metastatic patient. Ultimately, we hope these gains translate to a better quality of life for the patient with spinal metastases as these patients are still faced with incurable cancer.

The field of spine SRS is emerging. Significant research has been completed to guide the community with respect to evidence based inclusion and exclusion criteria [4], there have been scope of practice guidelines specific to spine SRS published by the Canadian Association of Radiation Oncology (CARO) SBRT task force [1], there are international consensus guidelines to assist in target volume delineation [5], and we are now learning more about the potential long-term complications of this treatment from the predominantly retrospective reviews of institutional experiences that adopted this technique early on in its development. Some of the critical areas of toxicity that patients would not otherwise be exposed to with conventional radiation, include a risk of radiation myelopathy [6-8], vertebral compression fracture (VCF) [9] and serious esophageal complications [10]. There are now evidence-based guidelines for spinal cord tolerance specific to spine SRS for both radiation naive and re-irradiated patients that can guide safe practice [6,7], we are learning about dose limits for the esophagus with single fraction SRS [10], and also predictors of VCF with respect to dosimetric and anatomic factors that may help select patients for stabilization prior to, or after, SRS [9]. Ultimately the patient can now be better informed as to the risks of spine SRS.

With respect to the current clinical evidence supporting efficacy, the indications for spine SRS can be broken down into those who are radiation naive, those with prior radiation requiring salvage SRS for tumor progression, and patients who are post-operative and require adjuvant radiotherapy [2]. The outcomes for each of these cohorts have been summarized in recent reviews and overall demonstrate efficacy $[2,11]$. Given that the field is still emerging and clinical trials are maturing, the high quality evidence required to be definitive in support of spine SRS is lacking, but a priority in the academic community at large. There is currently a randomized study comparing conventional radiation to SRS in patients who are radiation naive. The Radiation Therapy Oncology Group (RTOG) 0631 randomized study is testing 8 Gy in 1 fraction delivered with conventional radiation to $16-18$ Gy in 1 fraction using SRS.
There will be a need to conduct further studies to clarify the role of spine SRS in re-irradiation patients and post-operative SRS as compared to conventional radiation. Additionally, even in the radiation naive patient further studies will be required to test fractionated conventional radiation (for example, $30 \mathrm{~Gy}$ in 10 fractions) to higher dose spine SRS (for example, $24 \mathrm{~Gy}$ in 1 or 2 or 3 fractions). Once spine SRS is established as a standard of care, we will then need to organize dose-based randomized studies focused on SRS to determine what the optimal spine SRS therapy is (for example, single fraction SRS vs. fractionated SRS). There is conflicting data as to the benefits of high dose single fraction SRS to high dose fractionated SRS [12,13], and this is an important question that needs to be answered. Overall we are in a very challenging time in the age of spine SRS and the field requires significant international collaboration amongst both radiation oncologists and spine surgeons to move forward.

\section{References}

1. Sahgal A, Roberge D, Schellenberg D, Purdie TG, Swaminath A, et al. (2012) The Canadian Association of Radiation Oncology Scope of Practice Guidelines for Lung, Liver and Spine Stereotactic Body Radiotherapy. Clin Oncol (R Coll Radiol) 24: 629-639.

2. Sahgal A, Bilsky M, Chang EL, Ma L, Yamada Y, et al. (2011) Stereotactic body radiotherapy for spinal metastases: current status, with a focus on its application in the postoperative patient. J Neurosurg Spine 14: 151-166.

3. Sahgal A, Larson DA, Chang EL (2008) Stereotactic body radiosurgery for spinal metastases: a critical review. Int J Radiat Oncol Biol Phys 71: 652-665.

4. Lutz S, Berk L, Chang E, Chow E, Hahn C, et al. (2011) Palliative radiotherapy for bone metastases: an ASTRO evidence-based guideline. Int J Radiat Oncol Biol Phys 79: 965-976.

5. Cox BW, Spratt DE, Lovelock M, Bilsky MH, Lis E, et al. (2012) International Spine Radiosurgery Consortium consensus guidelines for target volume definition in spinal stereotactic radiosurgery. Int J Radiat Oncol Biol Phys 83 e597-e605.

6. Sahgal A, Ma L, Weinberg V, Gibbs IC, Chao S, et al. (2012) Reirradiation human spinal cord tolerance for stereotactic body radiotherapy. Int $\mathrm{J}$ Radiat Oncol Biol Phys 82: 107-116

7. Sahgal A, Weinberg V, Ma L, Chang E, Chao S, et al. (2012) Probabilities of radiation myelopathy specific to stereotactic body radiation therapy to guide safe practice. Int J Radiat Oncol Biol Phys.

8. Sahgal A, Ma L, Gibbs I, Gerszten PC, Ryu S, et al. (2010) Spinal cord tolerance for stereotactic body radiotherapy. Int J Radiat Oncol Biol Phys 77: 548-553.

9. Cunha MV, Al-Omair A, Atenafu EG, Masucci GL, Letourneau D, et al. (2012) Vertebral Compression Fracture (VCF) After Spine Stereotactic Body Radiation Therapy (SBRT): Analysis of Predictive Factors. Int J Radiat Oncol Biol Phys 84: e343-e349.

*Corresponding author: Arjun Sahgal, M.D. FRCPC, Department of Radiation Oncology, Princess Margaret Hospital and the Sunnybrook Health Sciences Centre, 5th Floor, 610 University Avenue, Toronto, Ontario, M5G2M9, Canada, Tel: 416-946-6513; Fax: 416-946-2227; E-mail: arjunsahgal@yahoo.com

Received October 15, 2012; Accepted October 15, 2012; Published October 17 2012

Citation: Sahgal A (2012) Current Status and the Future of Spine Radiosurgery. J Spine 1:e108. doi:10.4172/2165-7939.1000e108

Copyright: ( 2012 Sahgal A. This is an open-access article distributed under the terms of the Creative Commons Attribution License, which permits unrestricted use, distribution, and reproduction in any medium, provided the original author and source are credited. 
10. Cox BW, Jackson A, Hunt M, Bilsky M, Yamada Y (2012) Esophageal toxicity from high-dose, single-fraction paraspinal stereotactic radiosurgery. Int $J$ Radiat Oncol Biol Phys 83: e661-e667.

11. Masucci GL, Yu E, Ma L, Chang EL, Letourneau D, et al. (2011) Stereotactic body radiotherapy is an effective treatment in reirradiating spinal metastases: current status and practical considerations for safe practice. Expert Rev Anticancer Ther 11: 1923-1933.
12. Yamada Y, Bilsky MH, Lovelock DM, Venkatraman ES, Toner S, et al. (2008) High-dose, single-fraction image-guided intensity-modulated radiotherapy for metastatic spinal lesions. Int J Radiat Oncol Biol Phys 71: 484-490.

13. Jhaveri PM, Teh BS, Paulino AC, Blanco AI, Lo SS, et al. (2012) A doseresponse relationship for time to bone pain resolution after stereotactic body radiotherapy (SBRT) for renal cell carcinoma (RCC) bony metastases. Acta Oncol 51: 584-588. 